\title{
Technical challenge in MCA bifurcation aneurysm clipping
}

\author{
-Pankaj Raj Nepal ${ }^{1}$,FCPS \\ ${ }^{1}$ Head of Department, Department of Neurosurgery, B \& C Medical College \\ Teaching Hosptial, Birtamode, Jhapa.
}

Technical challenges in aneurysms can broadly be divided into general aneurysmal management challenges and aneurysm specific challenges. Specifically, we usually prefer to select the approach based on the presence or absence of temporal hematoma, and the length of the M1 segment. In the presence of temporal lobe hematoma it is wise to select the superior temporal gyrus approach, as hematoma evacuation is easy and we usually find the M3 segment to trace back to find the aneurysm. Other challenge lies while clipping the aneurysm. MCA bifurcation aneurysm seems to be the most notorious type, because of its varied morphology. We may find them a simple, multiple lobed, giant, fusiform, and occasionally its the M2 stuck to the neck of the aneurysm or arising from its dome. For multiple lobed MCA bifurcation aneurysm, the pearls of clipping is to think different lobes as a different aneurysms.

Keywords: Aneurysm, Microsurgical clipping, subarachnoid hemorrhage, middle cerebral artery

$\mathrm{T}$

echnical challenges in aneurysms can

broadly be divided into general aneurysmal management challenges and aneurysm specific challenges.

General challenges includes re- bleeding, vasospasm, hydrocephalus, and the medical complications like stunted myocardium, neurogenic pulmonary edema, ventilator associated pneumonia, ARDS, infective complications like urinary tract infection, other renal complications, and so on.

Re- bleeding is high with the proximal aneurysms, and the posterior circulating aneurysms. For predicting vasospasm, various articles are found in the literature, of all modified Fischer's grading is found to be more user friendly.
Here we are discussing the technical challenges we face in the setting of ruptured MCA bifurcation aneurysms.

Preoperative challenge: managing the blood pressure, and intracranial pressure is the technical challenge.

Intraoperative challenge: we usually prefer to select the approach based on the presence or absence of temporal hematoma, and the length of the M1 segment .

In the presence of temporal lobe hematoma it is wise to select the superior temporal gyrus approach, as hematoma evacuation is easy and we usually find the M3 segment to trace back to find the aneurysm. 


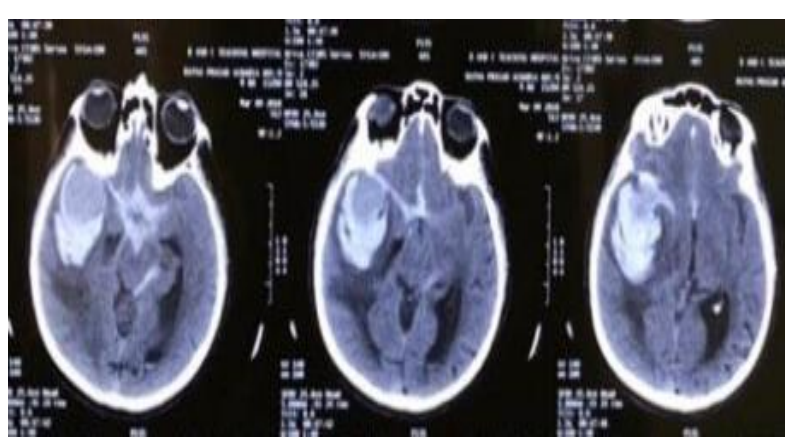

Figure 1: Giant MCA aneurysm with temporal lobe hematoma

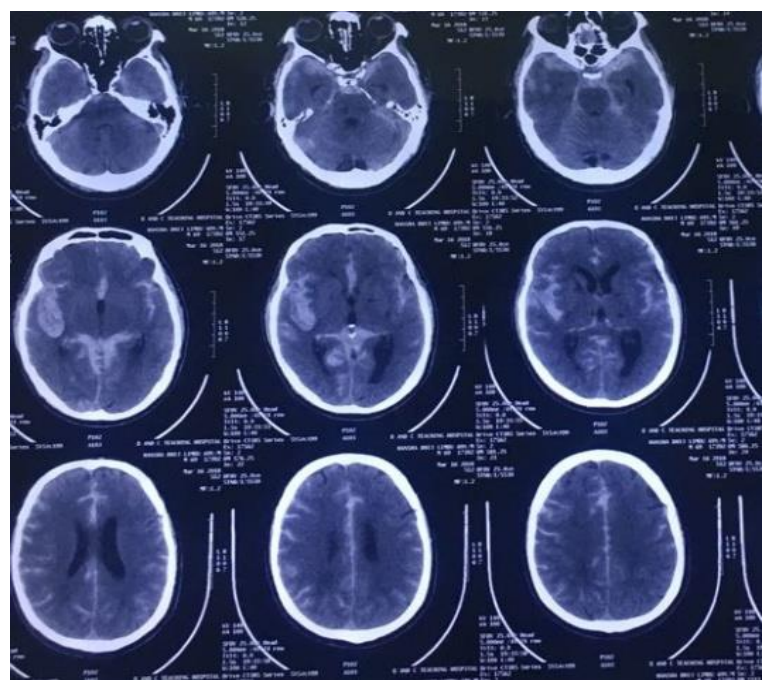

Figure 3:Diffuse subarachnoid hemorrhage

Diffuse subarachnoid hemorrhage in ruptured MCA bifurcation aneurysms, trans- sylvian approach is considered the best, which is further seen as proximal to distal dissection or distal to proximal dissection. We prefer to select the approach based on the length of the M1 segment. For cases of short M1 segment proximal to distal and for longer M1 segment we go distal to proximal approach.

like in hematoma case we went superior temporal gyrus approach and in diffuse SAH case we went transsylvian approach.

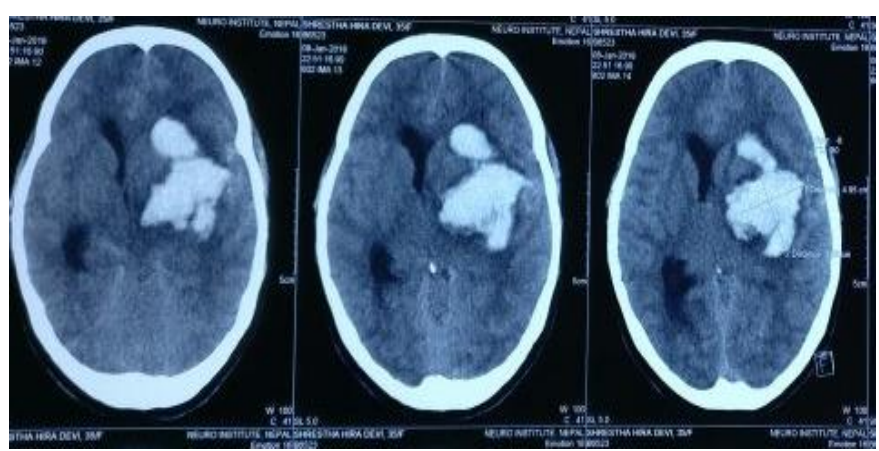

Figure 2: Left MCA bifurcation aneurysm presented with left temporal bleed extending to left putamen

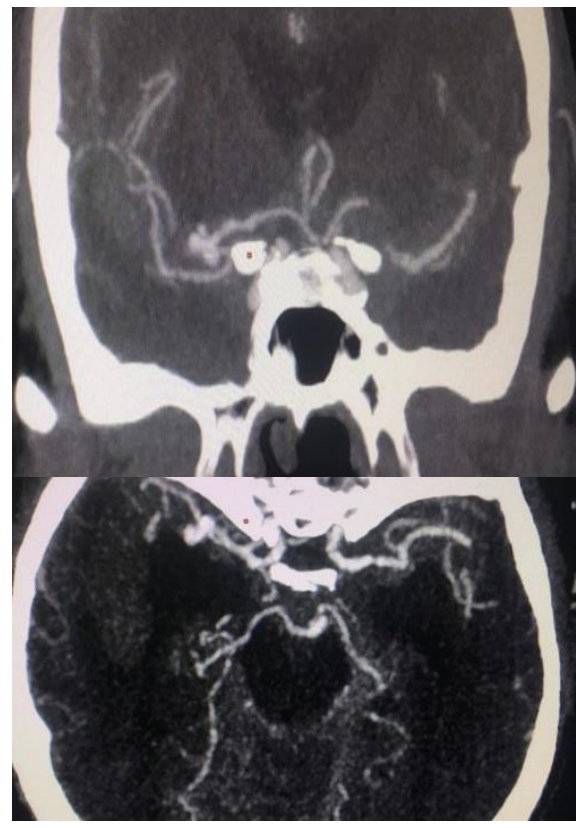

Figure 4: CT angiogram with right MCA bifurcation aneurysm with short M1 segment 
These angiographies had the short M1 segments so we went the proxima to distal sylvian dissection and traced back the aneurysm from the M1 segment.

\section{Challenge during clipping MCA} bifurcation aneurysm: Other challenge lies while clipping the aneurysm. MCA bifurcation aneurysm seems to be the most notorious type, because of its varied morphology. We may find them a simple, multiple lobed, giant, fusiform, and occasionally its the M2 stuck to the neck of the aneurysm or arising from its dome.

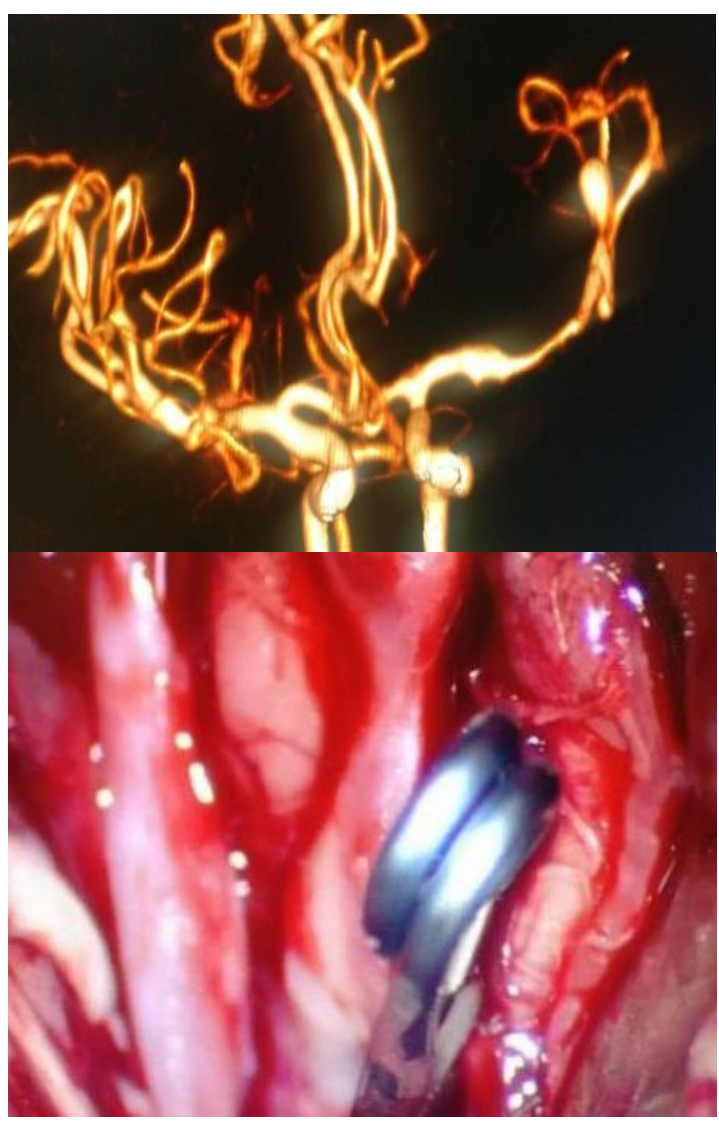

\section{Figure 5: Simple clipping technique}

Simple aneurysms could be clipped easily with a straight or angled clips. Here in the above case it was the superior pointing simple MCA bifurcation aneurysm and was clipped with a straight clip.

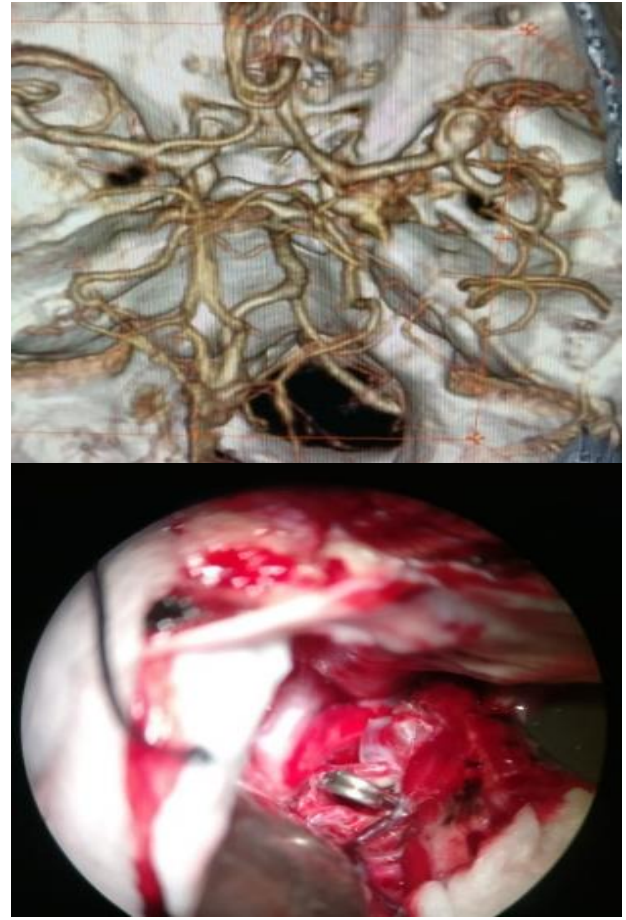

Figure 6: Simple clipping with angled clip

Similarly, this is an another example of a simple aneurysm with its dome pointing inferior. In this case the clipping was amenable with an angled clip. 


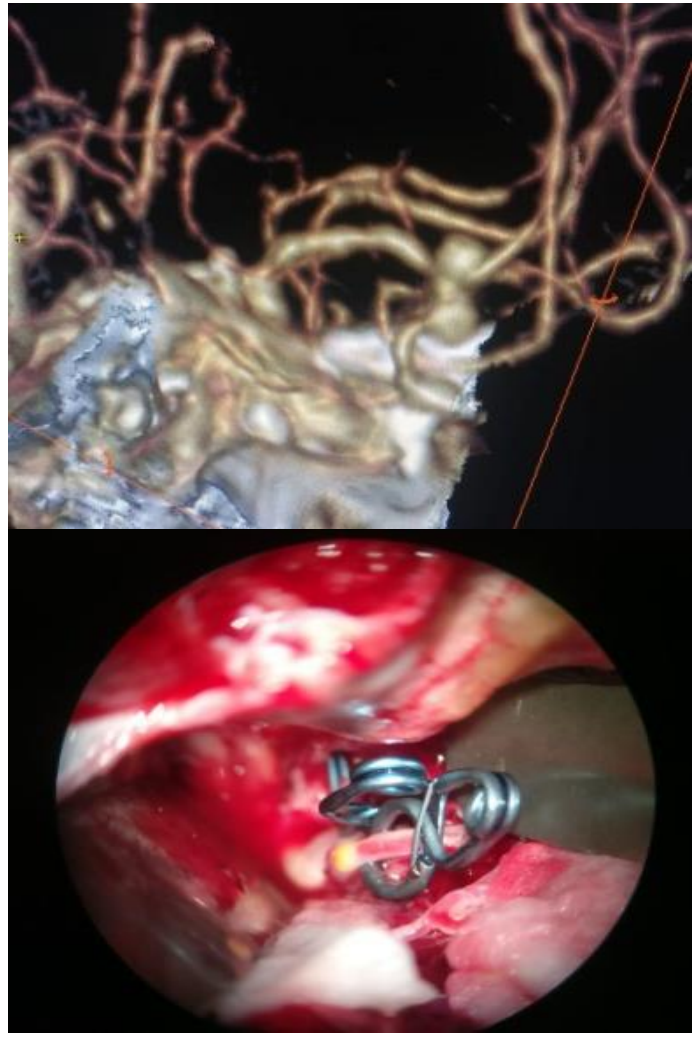

Figure 7: Modified clipping technique for clipping multilobed aneurysm

For multiple lobed MCA bifurcation aneurysm, the pearls of clipping is to think different lobes as a different aneurysms. In the above case, there was superior pointing lobe and inferior pointing lobe. Inferior pointing lobe was clipped with a simple angled clip, however while clipping the superior pointing lobe the M2 was in our way, which made us to use the angled fenestrated clip. It is always required to secure the distal circulation in the M2, for which either use of ICG dye or visually confirming the patency of the vessel and making sure it is not taken in the tip of the clips is required.

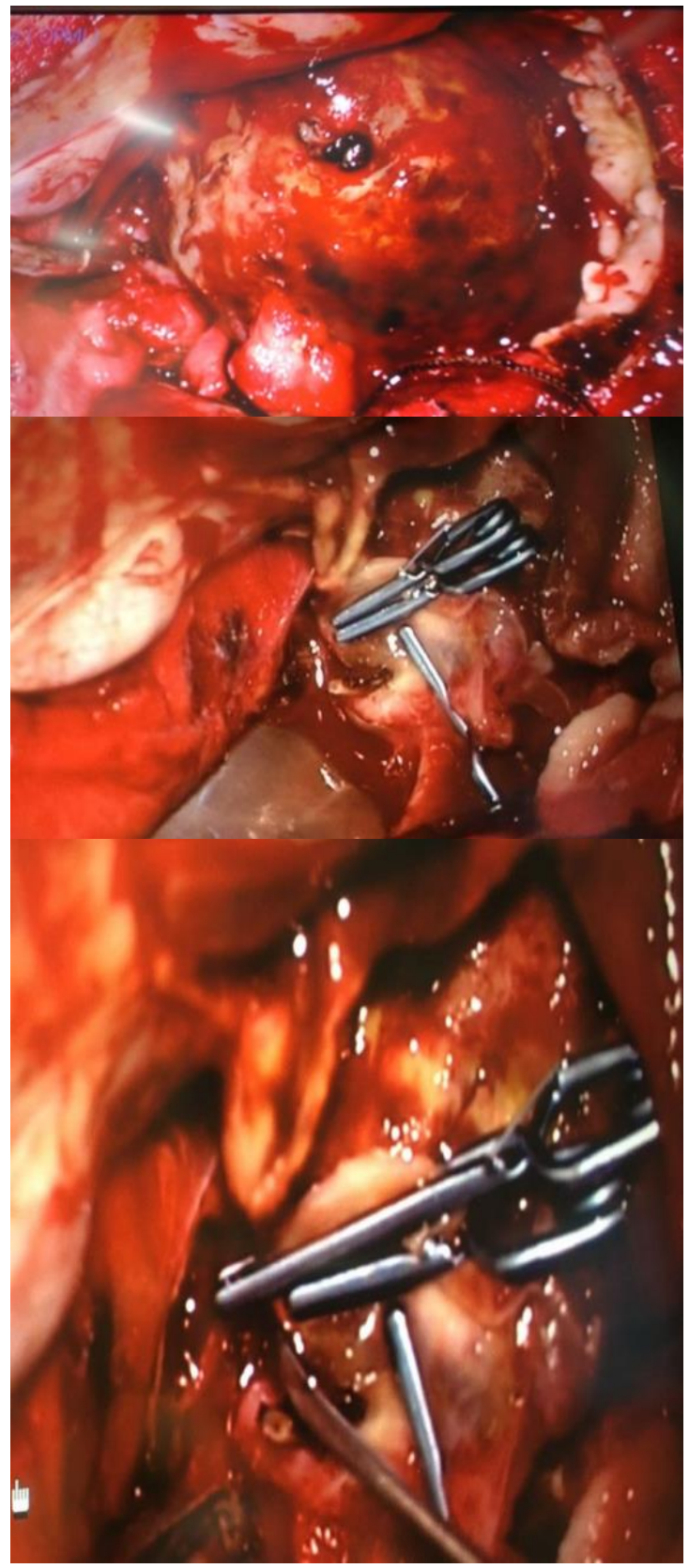

Figure 8: Partial excision and clip reconstruction for giant aneurysm

For Giant MCA bifurcation aneurysm, various technique of clipping is described. The pearls in its clipping is in creating a secured flow from the M1 to M2 vessels. If we study our angiography very carefully, we usually find the vessels are at the neck of the aneurysm and its the dome which has ruptured. In this setting tandam clipping or clipping with intersecting clips could on the fundus with 
creating the distal circulation by leaving the cuff of tissue over the neck could be very effective.

But occasionally we might find a case like ours, where the giant aneurysm was completely thrombosed and hard, and blood found some way through the aneurysm to get ruptured through the fundus. In such setting no clips application will work without removing the thrombus. So, we performed the partial excision of the wall of the aneurysm, and excision of the thrombus inside and then clip reconstruction. Three clips were used two were stacked and one long angled to occlude the aneurysm. In this case we had to exchange the outermost stacked clip with a longer clip to completely occlude the aneurysm and the distal circulation was made patent.

\section{References:}

1. Devkota UP, Aryal KR. Result of surgery for ruptured intracranial aneurysms in Nepal. Br J Neurosurg 15(1): 13-6, 2001

2. Fox J. Intracranial Aneurysms. New York: Springer-Verlag;1983.

3. Kassell NF , Torner JC, Haley EC Jr , Jane JA, Adams HP , Kongable GL . The International Cooperative Study on the Timing of Aneurysm Surgery. Part 1: overall management results . J Neurosurg 73:18 - 36, 1990

4. Molyneux A , Kerr R ; International Subarachnoid Aneurysm Trial (ISAT) Collaborative Group, Stratton I, Sandercock P, Clarke M, Shrimpton J , Holman R . International Subarachnoid Aneurysm Trial (ISAT) of neurosurgical clipping versus endovascular coiling in 2143 patients with ruptured intracranial aneurysms: a randomized trial . J Stroke Cerebrovasc Dis 11:304 - 314, 2002

5. Molyneux A, Kerr R ; International Subarachnoid Aneurysm Trial (ISAT) Collaborative Group , Stratton I , Sandercock P , Clarke M , Shrimpton J , Holman R . International Subarachnoid Aneurysm Trial (ISAT) of neurosurgical clipping versus endovascular coiling in 2143 patients with ruptured intracranial aneurysms: a randomized trial . J

Stroke Cerebrovasc Dis 11:304 - 314, 2002 
6. Roka YB, Shrestha M, Puri PR, Adhikari HB. Surgery for Intracerebral Aneurysms in Eastern Nepal: A New Beginning. Nepal Journal of Neuroscience 9:5-9, 2012

7. Sharma GR, Kausal P, Jha R, Khadka N, Adhikari DR, Bista P, Sultania PK. Outcome of Microsurgical Clipping of Intracranial Aneurysms in Bir Hospital. Nepal Journal of Neuroscience 8(2): 137-142, 2011

8. Wolstenholme J , Rivero-Arias O , Gray A , Molyneux AJ , Kerr RS , Yarnold JA, Sneade M ; International Subarachnoid Aneurysm Trial (ISAT) Collaborative Group . Treatment pathways, resource use, and costs of endovascular coiling versus surgical clipping after a SAH . Stroke 39:111 - 119, 2008 